\title{
A Panel of Four-IncRNA Signature as a Potential Biomarker for Predicting Survival in Clear Cell Renal Cell Carcinoma
}

\author{
Haoran Liu ${ }^{1,2 \#}$, Tao Ye ${ }^{1,2 \#}$, Xiaoqi Yang1,2, Peng Lv¹,2, Xiaoliang $\mathrm{Wu}^{1,2}$, Hui Zhou ${ }^{1,2}$, Jin Zeng1,2, Kun \\ Tang $1,2, \bowtie$, Zhangqun Ye ${ }^{1,2}$ \\ 1. Department of Urology, Tongji Hospital, Tongji Medical College, Huazhong University of Science and Technology, Wuhan 430030, China \\ 2. Hubei Institute of Urology, Wuhan 430030, China \\ \#Haoran Liu and Tao Ye contributed equally to this work.
}

$\square$ Corresponding author: Professor Kun Tang, Department of Urology, Tongji Hospital, Tongji Medical College, Huazhong University of Science and Technology, Institute of Urology of Hubei Province, Wuhan 430030, China. Email: tangsk1990@163.com

() The author(s). This is an open access article distributed under the terms of the Creative Commons Attribution License (https://creativecommons.org/licenses/by/4.0/). See http://ivyspring.com/terms for full terms and conditions.

Received: 2019.09.18; Accepted: 2020.03.06; Published: 2020.04.27

\begin{abstract}
Long non-coding RNAs (IncRNAs) have been considered as biomarkers for the carcinogenesis and development of various cancers. However, the prognostic significance of IncRNAs in renal cell carcinoma (RCC) remains unclear. This study aimed to determine the predictive ability of IncRNAs in clear cell RCC (ccRCC). Among the cohort of kidney renal clear cell carcinoma (KIRC) of the The Cancer Genome Atlas (TCGA), 525 patients were enrolled in our study. Expression of IncRNAs based on RNAseq was obtained from TCGA. Kaplan-Meier prognostic analysis and a Cox proportional hazards regression model were used to assess related factors. The IncRNA signature was then validated in an independent cohort of an additional $60 \mathrm{ccRCC}$ patients. Hierarchical clustering of the KIRC TCGA dataset identified 26 differentially expressed IncRNAs (11 down-regulated and 15 up-regulated) using average linkage clustering. Kaplan-Meier survival analysis identified 30 statistically significant IncRNAs that strongly predicted prognosis, with 4 ccRCC-specific IncRNAs (TCL6, PVT1, MIR155HG, and HARIB) being differentially expressed and correlating significantly with OS. Patients assigned to the high-risk group were associated with poor OS compared with patients in the low-risk group $(\mathrm{HR}=2.57 ; 95 \% \mathrm{Cl}, 1.89-3.50 ; \mathrm{p}<0.001)$. This finding was validated in the Tongji Hospital cohort, and the four-IncRNA signature was shown to be significantly predictive of $c c R C C$ prognosis $(p<0.001)$. In this study, we constructed an applicable four-IncRNA-based classifier as a reliable prognostic and predictive tool for OS in patients with ccRCC.
\end{abstract}

Key words: IncRNAs; Prognosis; Survival; Clear Cell Renal Cell Carcinoma

\section{Introduction}

Currently, renal cell carcinoma (RCC) has become the most general malignant tumor of the kidneys in adults, corresponding to $3.7 \%$ of all adult cancers worldwide. RCC is also an important cause of cancer-related morbidity and mortality globally [1]. Clear cell renal cell carcinoma (ccRCC) is the most universal subtype, and it is very important to demonstrate the molecular changes linked to malignant transformation and longer survival [2]. The current pathological grade system and tumor node metastasis (TNM) stage of the American Joint Committee on Cancer (AJCC) exhibits valuable but insufficient prediction of prognosis and estimation for subsets of RCC patients [3]. Overall, clinicopathological risk factors limit their clinical application, without a clear prediction of disease 
recurrence, chemotherapy response or survival. As an increasing amount of evidence has demonstrated that the discovery and application of molecular biomarkers contributes to prognostic evaluation and identification of potential high-risk RCC patients [4], there is an increasing need to add new prognostic and predictive biomarkers to complement and improve the staging system currently in use. Such markers may also serve as therapeutic targets.

Long noncoding RNAs (lncRNAs), a class of noncoding RNAs, are identified as non-proteincoding transcripts of more than 200 nucleotides [5]. Since our previous study on the expression patterns of genome-wide lncRNAs in RCC based on microarray, increasing evidence has demonstrated that by acting as tumor suppressors or onco-lncRNAs, aberrant expression of lncRNAs plays a vital role in the development and evolution of many types of human carcinomas. Indeed, these molecules are important in regulating multiple and complex biological processes, for instance, cell proliferation, metabolism, differentiation, angiogenesis and the epithelialmesenchymal transition (EMT) [6]. Recently, many studies have explored the value of lncRNAs as minimally invasive biomarkers for diagnosis, prognosis or monitoring curative effects in various cancers, including ccRCC [7-10]. Nonetheless, more potential and valuable lncRNA biomarkers are needed to improve the clinical outcomes of ccRCC patients.

Our present study was designed to demonstrate the prognostic potential of lncRNAs in ccRCC and identify a potential panel of four-lncRNA signatures as a composite biomarker for risk stratification of ccRCC patients to complement traditional clinicopathological prognostic factors. The signature developed can help stratify ccRCC patients for optimal treatment strategies.

\section{Materials and methods}

\section{Expression profiles and sample information}

We downloaded the RNAseq data from TCGA, mainly containing the lncRNA dataset (Level 3) and clinical data for RCC patients based on kidney renal clear cell carcinoma (KIRC) samples using the Illumina HiSeq 2000 platform. Our study was in accord with TCGA publication guidelines. In total, 525 primary ccRCC tumor samples with detailed lncRNA expression data were collected from the current TCGA database, and the process applied met with the parameters identified in the initial-phase study [11]. The patients met the following criteria: fully characterized tumors, complete OS data and RNAseq information and no pretreatment. We collected the clinicopathological characteristics of the included patients with RCC, mainly including age, gender, tumor size, laterality, TNM, tumor grade, tumor stage, and overall survival. The primary end-point in our study was OS. Extended demographic parameters of the patients, as characterized by TCGA consortium, are presented in Table S1 of Additional file 2. Because the data we collected were retrieved from TCGA, the conventional additional approval by an ethics committee was not necessary. Data processing was conducted according to TCGA human subject protection and data access policies.

For our Tongji Hospital cohort, 60 pairs of RCC and adjacent nontumor tissues were collected from patients who underwent radical nephrectomy in our hospital from December 2015 to January 2019. Approval of the Ethical Committee of Tongji Hospital was obtained, and all patients included were fully informed of our study. All of the tissue samples were collected during surgery and preserved at $-80^{\circ} \mathrm{C}$. Two experienced pathologists confirmed the pathological subtypes. Every sample was instantly frozen in liquid nitrogen and preserved at $-80^{\circ} \mathrm{C}$ for later RNA extraction and qPCR analysis. All clinicopathological characteristics were obtained from electronic records, mainly including age, gender, tumor size, tumor position, tumor stage, TNM, and tumor grade. Follow-up was conducted quarterly by telephone or in the clinic. Significant survival events, including tumor progression, recurrence, metastasis and death, were recorded. Samples were obtained under informed consent and approval of the Ethics Committees of Tongji Hospital.

\section{Cluster analysis of datasets}

Hierarchical cluster analysis was employed to explore relationships among the results of individual studies. The overall rank matrix was established on the basis of rank matrices acquired from independent analysis of up-regulated and down-regulated lncRNA profiles. Within the matrix, lncRNAs with a value of 0.5 are not reported in this study. LncRNAs with a value greater than 0.5 were considered to be up-regulated (one minus normalized rank of lncRNA from the analysis of lncRNA lists), and those values less than 0.5 were considered to be down-regulated (normalized rank as the outcome of analysis for down-regulated lncRNA lists). Spearman rank correlation combined with average linkage method was utilized in the cluster analysis.

\section{RNA extraction and qPCR}

Total RNA was extracted from the frozen samples using TRIzol (Invitrogen) according to the 
manufacturer's protocol. Oligo-dT primers and superscript II reverse transcriptase (Invitrogen) were utilized to generate first-strand complementary DNA (cDNA). After that, a quantification trial of four deregulated lncRNAs was conducted by qPCR using SYBR Premix ExTaq with an MX3000. U6 primers were acquired from GeneCopoeia. qPCR was conducted under the following protocol: $95^{\circ} \mathrm{C}$ for 10 min, followed by 40 cycles of $95^{\circ} \mathrm{C}$ for $10 \mathrm{sec}, 60^{\circ} \mathrm{C}$ for $20 \mathrm{sec}$ and $72^{\circ} \mathrm{C}$ for $30 \mathrm{sec}$, with $95^{\circ} \mathrm{C}$ for $1 \mathrm{~min}$ and $60^{\circ} \mathrm{C}$ for $1 \mathrm{~min}$. Additionally, every trials were accomplished in triplicate. Expression levels were standardized to GAPDH. The relative fold-changes of lncRNA expression were calculated using the ${ }^{\Delta} \mathrm{CT}$ method, and the outcomes were expressed as $2{ }^{-} \Delta \mathrm{C}$ T. The lncRNA primers used for qPCR validation in this study are listed in Table S3 of Additional file 2.

\section{Statistical analysis}

OS was defined as from the day of diagnosis to the day of death or final follow-up. Patients with no events or still alive until the day of the final follow-up were censored. Statistical analysis was performed by SPSS 17.0 software, and survival curves were drawn using the Kaplan-Meier method with log-rank tests to evaluate differences between groups. LncRNAs shown to be differentially expressed $(F C>1.66 \& F C<0.60)$ were further studied. Univariate Cox regression analysis was employed to estimate correlations between the overall survival of ccRCC patients and expression level of each lncRNA. Hazard ratios (HR) and 95\% confidence intervals (CI) were obtained. A total of four lncRNAs were identified and separated into two parts, a high-risk group and a low-risk group, using the median of the discovery series as the cut-off point. A risk score formula to predict OS was generated on the basis of a linear combination of the expression level multiplied by the regression coefficient obtained from the Univariate Cox regression model $(\beta)$ : risk score = explncRNA1* $\beta$ lncRNA1 + explncRNA2* $\beta \operatorname{lncRNA2}+$ $\ldots$ explncRNAn* ${ }^{*} \ln \mathrm{R} R \mathrm{NAn}$. By utilizing the median risk score as the cut-off, the included ccRCC patients were separated into high-score and low-score groups. A four-lncRNA expression signature was then constructed using a linear combination of the expression levels of the four IncRNAs and the estimated regression confidence interval in the multivariate Cox regression analysis, as previously described. Kaplan-Meier survival curves with the log-rank test were used to evaluate differences in OS between the two groups with high-risk and low-risk lncRNAs. Univariate and multivariate Cox proportional hazards analyses of lncRNA expression and ccRCC patient OS in TCGA were also analyzed. A two-sided $\mathrm{p}$ value less than 0.05 was identified as statistically significant.

\section{Results}

\section{CCRCC patient features in TCGA and the Tongji Hospital validation cohort}

A total of 525 ccRCC patients with a median age of 61 from TCGA were enrolled for analysis in our study. Additionally, $60 \mathrm{ccRCC}$ patients with a median age of 58.6 from the Tongji Hospital cohort were included. The clinical characteristics of the two ccRCC cohorts, including gender, tumor size, TNM stage, tumor grade, laterality, lymph node status and metastasis, are shown in Table S1 of Additional file 2. The median follow-up time was 79.5 months and 52.5 months for the cohorts from TCGA and Tongii, respectively.

\section{Screening of differentially expressed IncRNAs in CCRCC patients}

According to our exclusion criterion, lncRNAs that were differentially expressed $(\mathrm{FC}>1.66 \& \mathrm{FC}<0.60)$ were further studied. A total of 26 lncRNAs (11 downregulated, UCA1; C15orf2; LOC728606; TCL6; LOC554202; TERC; TRPM3; ASFMR1; RMST; SEMA3G and CASC2, and 15 upregulated, PVT1; PTHLH; PSORS1C3; HAR1B; DGCR5; MIR155HG; XIST; MIAT; HAR1A; SNHG4; SNHG3; PRINS; HOTAIR; DLEU2 and MIR17HG; Figure 1, Table S2 of Additional file 2) were differentially expressed in the ccRCC patients.

\section{Screening of IncRNAs significantly associated with overall survival in CCRCC patients}

Using the median value of lncRNA expression as a cut-off point, we further performed the Kaplan-Meier overall survival analysis for each lncRNA in ccRCC patients using the dataset from TCGA (Figure 2). A total of 30 lncRNAs (7 protective lncRNAs, WRAP53, TRPM3, TCL6; SEMA3G, CCND1 and DAPK1; and 23 potentially risk lncRNAs, SNHG3, MIAT, HOTAIR, MINA, HAR1A, DISC2, SRA1, SNHG11， DMPK, PVT1; MIR155HG, KCNQ1OT1, DLEU2, HYMAI, PRINS, MEG3, RRP1B, SNHG4, MALAT1 GAS5, FADS1, HAR1B and PCGEM1; Figure S1 of Additional file 1) were validated to be significantly related to overall survival in the ccRCC patients.

\section{Identification of the four-IncRNA signature}

Combined analysis of ccRCC-specific lncRNAs, which were both differentially expressed (Figure 2; $F C>1.66 \& F C<0.60$ ) and significantly associated with overall survival (Table S2), was performed. Finally, we identified a four-lncRNA signature including one 
downregulated (TCL6) and three upregulated (PVT1, MIR155HG, and HAR1B) lncRNAs using the dataset from TCGA (Table S4 of Additional file 2). As based on Cox proportional hazards analysis, low expression of TCL6 (HR: 0.48, 95\%CI: 0.36-0.66) with high expression of PVT1 (HR: 1.79, 95\%CI: 1.32-2.43), MIR155HG (HR: 1.76, 95\%CI: 1.30-2.39) and HAR1B (HR: 1.56, 95\%CI: 1.14-2.13) was associated with poor overall survival (Figure 3 ).

\section{The IncRNA signature risk score as an independent indicator for CCRCC prognosis}

A risk-score formula was created based on the expression of these four lncRNAs for OS prediction, as follows: Risk score $=\left(0.167^{*}\right.$ expression level of PVT1 $)+\left(0.149^{*}\right.$ expression level of MIR155HG $)+$ (0.162* expression level of HAR1B)- $\left(0.109^{*}\right.$ expression level of TCL6). The risk score based on the four lncRNAs was calculated for each ccRCC patient. By applying the median as the cut-off, 525 ccRCC patients were classified into a high-score group or a low-score group. The protective lncRNAs exhibited high expression in the low-score group, whereas the risk lncRNAs showed low expression in the high-score group (Figure 4, Figure S2 of Additional file 1). The ccRCC patients in the high-score group experienced a significantly worse OS (HR: 2.57, $95 \% \mathrm{CI}:$ 1.89-3.50, $\mathrm{p}<0.001)$ than those in the low-score group (Figure 4F). When we further performed Kaplan-Meier overall survival analysis of subgroups according to tumor grade (Figure S3 of Additional file 1) and stage (Figure S4 of Additional file 1) using the KIRC dataset from TCGA, the lncRNA signature risk score remained as a significant predictor for ccRCC overall survival.

Moreover, univariate Cox regression analyses showed that age $(p<0.001)$, laterality $(p=0.011)$, tumor size $(p<0.001)$, tumor TNM stage $(p<0.001)$, lymph node positivity $(\mathrm{p}=0.024)$, metastasis $(\mathrm{p}<$ $0.001)$ and risk score $(p<0.001)$ were significantly related to the overall survival of the ccRCC patients; multivariate Cox regression analysis revealed that age $(\mathrm{p}<0.001)$, tumor stage $(\mathrm{p}<0.001)$ and risk score $(\mathrm{p}<$ 0.001 ) were independent prognostic factors (Table 1 ).

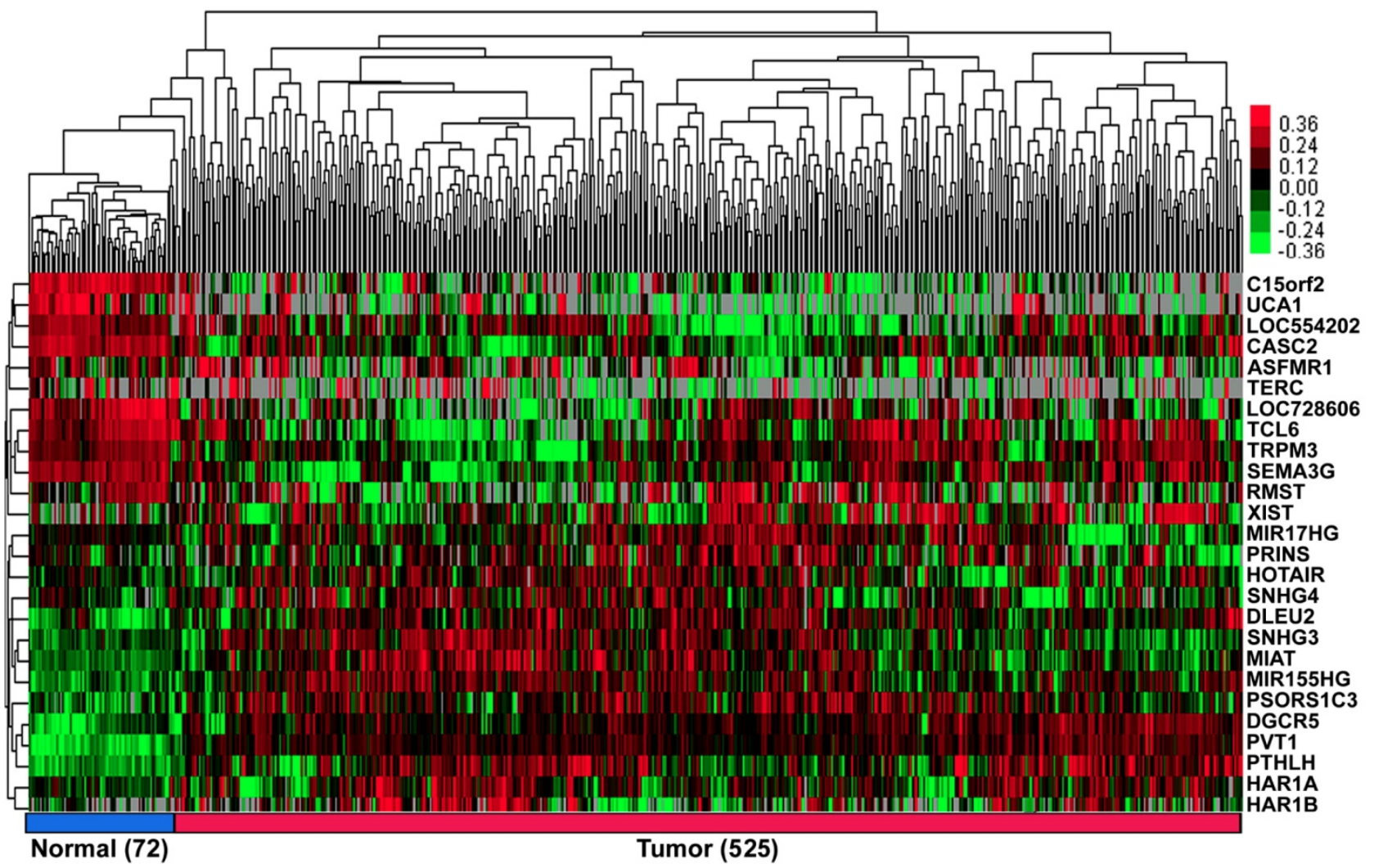

Figure 1. The heat map shows the relative fold change of IncRNAs in ccRCC compared with normal adjacent tissues. Hierarchical clustering of the KIRC TCGA dataset with 26 differentially expressed IncRNAs ( 11 down-regulated and 15 up-regulated) by average linkage clustering. Each row represents a single IncRNA, and each column represents a single sample. Pseudocolours show transcript levels from low to high on a log 2 scale from -3 to 3 , ranging from a low (dark, black) to a high (bright, red, or green) association. Short red and green vertical bars indicate upregulated and downregulated IncRNAs, respectively. The black bar with the pseudocolour 0 indicates no signal in the RNAseq data. 


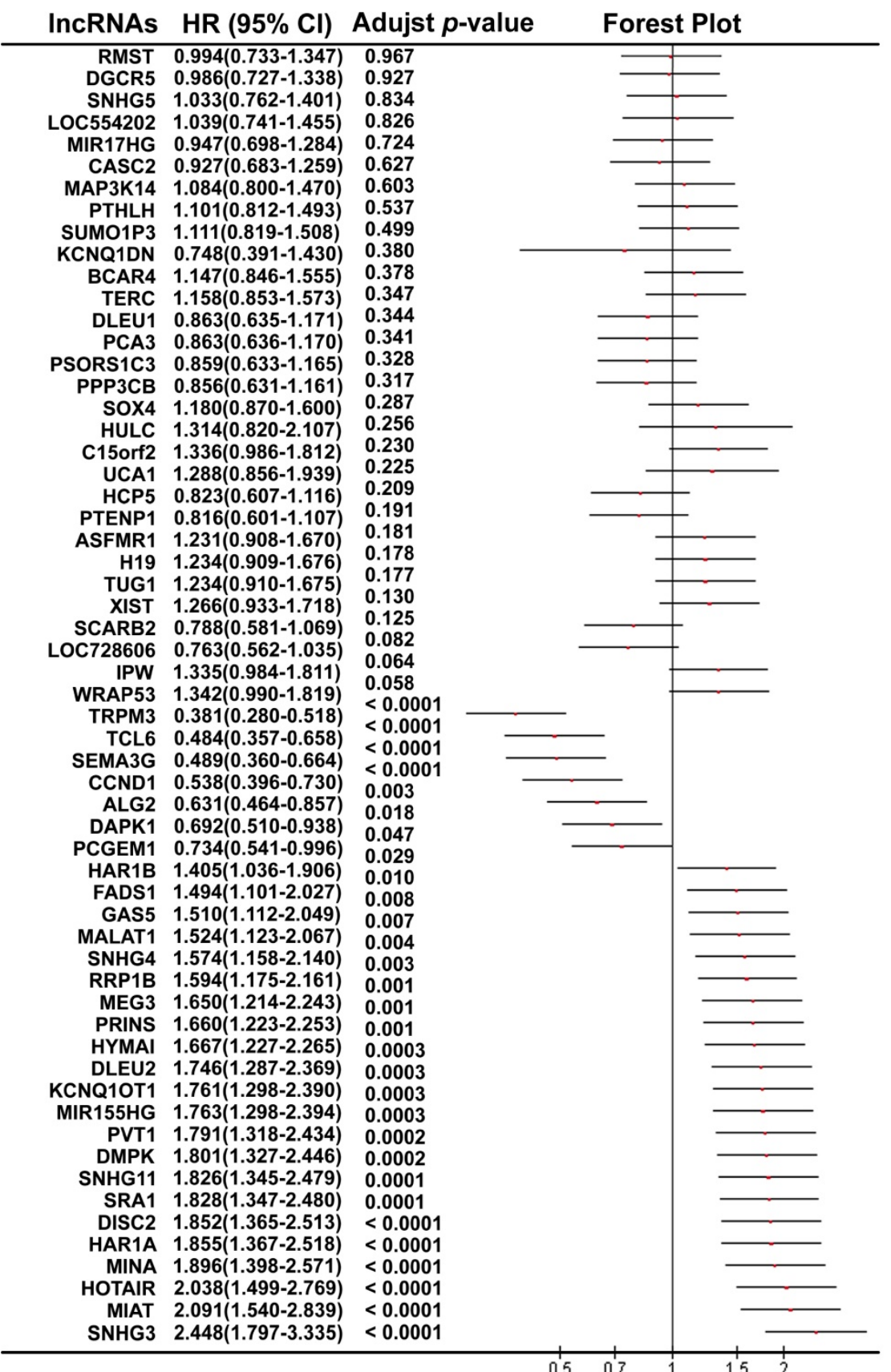

Figure 2. The forest plot shows the association between each IncRNA and OS in ccRCC patients using TCGA.

The IncRNA signature is only specific for CCRCC

Although we constructed a promising 4-lncRNA panel for ccRCC prognosis, it is uncertain if this panel is only specific for ccRCC. Thus, additional studies were performed to further examine the changes in expression of these four lncRNAs using kidney renal papillary cell carcinoma (KIRP) and kidney chromophobe (KICH) databases. According to Kaplan-Meier curve analysis, the 4-lncRNA signature could not predict overall survival in KIRP and $\mathrm{KICH}$ (Figure S5 of Additional file 1).
Confirmation of the expression and prognostic value of the top four dysregulated IncRNAs in Tongji cohort ccRCC patients

Considering the basis of the lncRNA profiling outcomes in TCGA, we further detected ccRCC-related lncRNA expression using qPCR to examine $60 \mathrm{ccRCC}$ samples from Tongji Hospital to estimate and validate the value of the candidate lncRNAs for prognosis. We selected the panel of four lncRNAs for this qPCR verification analysis. The expression level of TCL6 was decreased whereas the levels of PVT1, MIR155HG and HAR1B were 
increased in ccRCC tissues compared with adjacent normal tissue (all $p<0.01$ ). Similar results for expression of these four lncRNA were obtained when detected in four RCC cell lines compared with HK2 cells. The same prognostic score formula acquired from the dataset from TCGA was utilized to calculate the four-lncRNA signature score for each of the 60 patients in our validation cohort. Using the median value as the optimum cut-off point, we validated the four-signature lncRNAs as a potential prognostic biomarker (HR: 6.25, 95\% CI: 2.75-14.2, p < 0.001) (Figure 5).

\section{Discussion}

Many lncRNAs with abnormal expression that is highly related to different cancer types have been identified through genome-wide transcriptome analyses [8]. A series of studies have revealed that lncRNAs can act as regulators of diverse biological functions, including X-chromosome silencing, transcription regulation, and genomic stability [12, 13]. Recently, some studies have evaluated the prognostic relevance of lncRNAs in ccRCC patients,
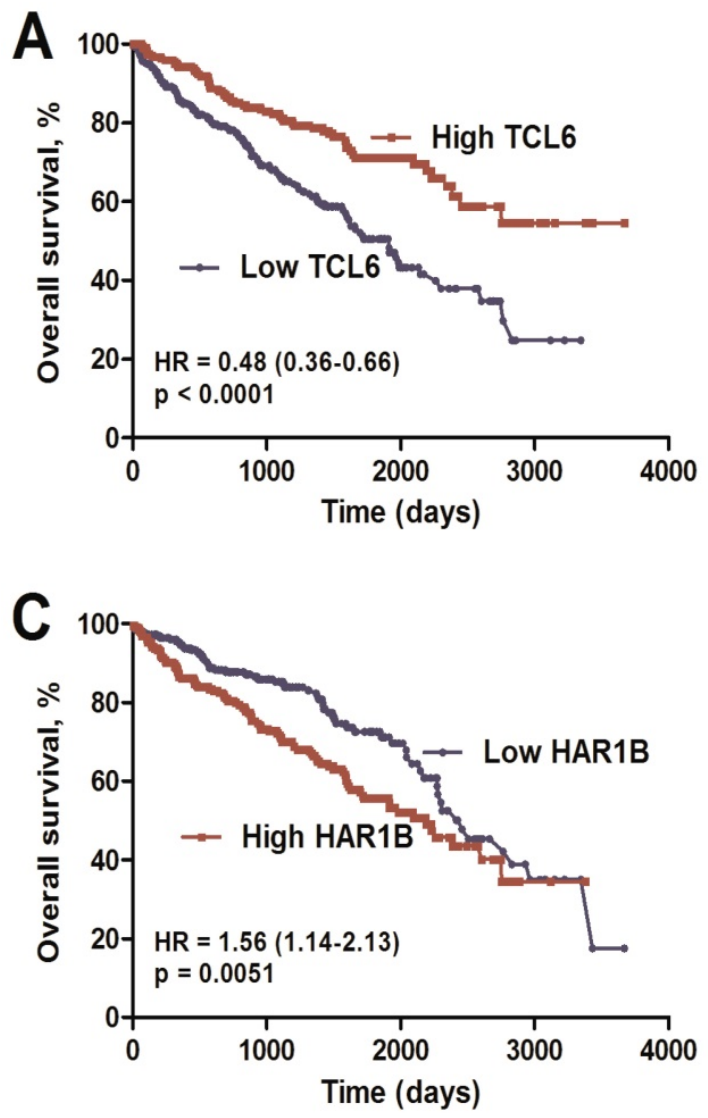

though most have focused on limited lncRNAs with a small number of patients. Therefore, lncRNA signatures might have concrete predictive and prognostic value in the management of RCC. The purpose of our study was to identify a lncRNA signature using TCGA data that is able to predict prognosis in RCC. A total of 525 RCC patients with corresponding clinical data were enrolled, and lncRNAs significantly related to overall survival (OS) in RCC patients were assessed in a Cox proportional regression model. We generated a risk-score formula to examine the value of the lncRNA signature in predicting RCC prognosis. Four lncRNAs were confirmed to be markedly related to OS in RCC patients. Patients with high risk scores experienced lower overall survival than patients who had low risk scores, and multivariate Cox regression analyses showed that the IncRNA signature could perform independently as an indicator of prognosis. In addition, the signature was validated as a predicator in our Tongji ccRCC cohort. In summary, our study identified a 4-lncRNA signature that could serve as an independent marker in the prognosis of ccRCC.
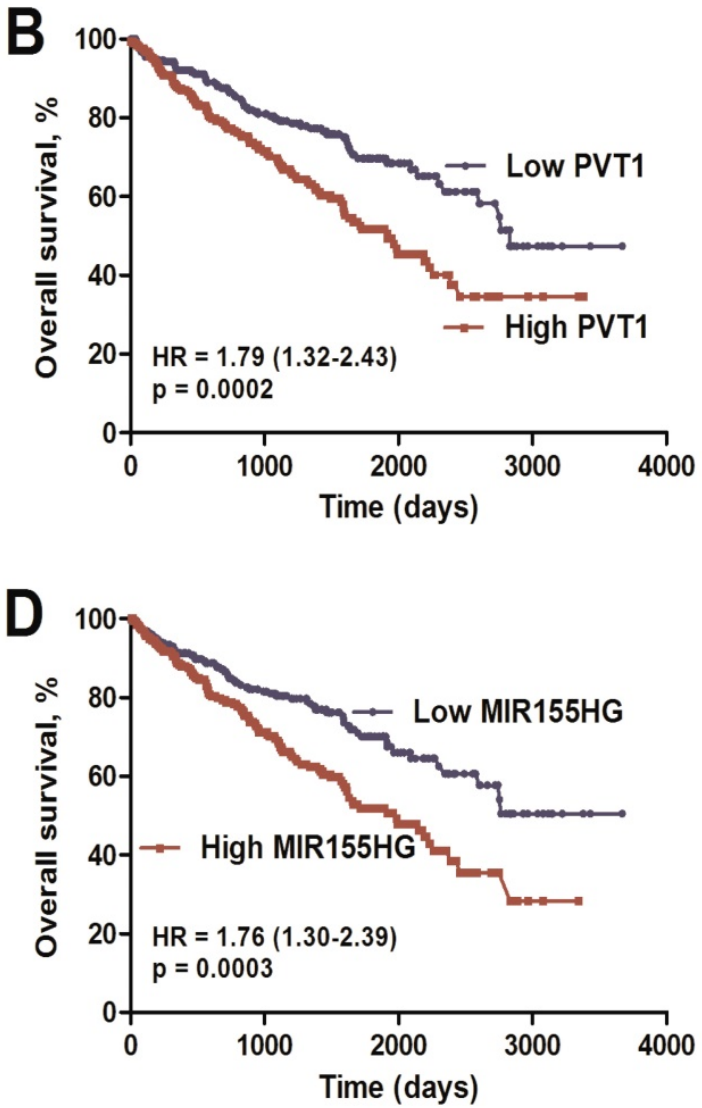

Figure 3. Kaplan-Meier overall survival analysis of the four IncRNAs using the KIRC TCGA dataset. Kaplan-Meier plots of overall survival in the KIRC TCGA cohort are shown according to IncRNA expression (TCL6 (A), PVT1 (B), MIR155HG (C), HAR1B (D)). The relative median expression value of each IncRNA was used as the cut-off point. 


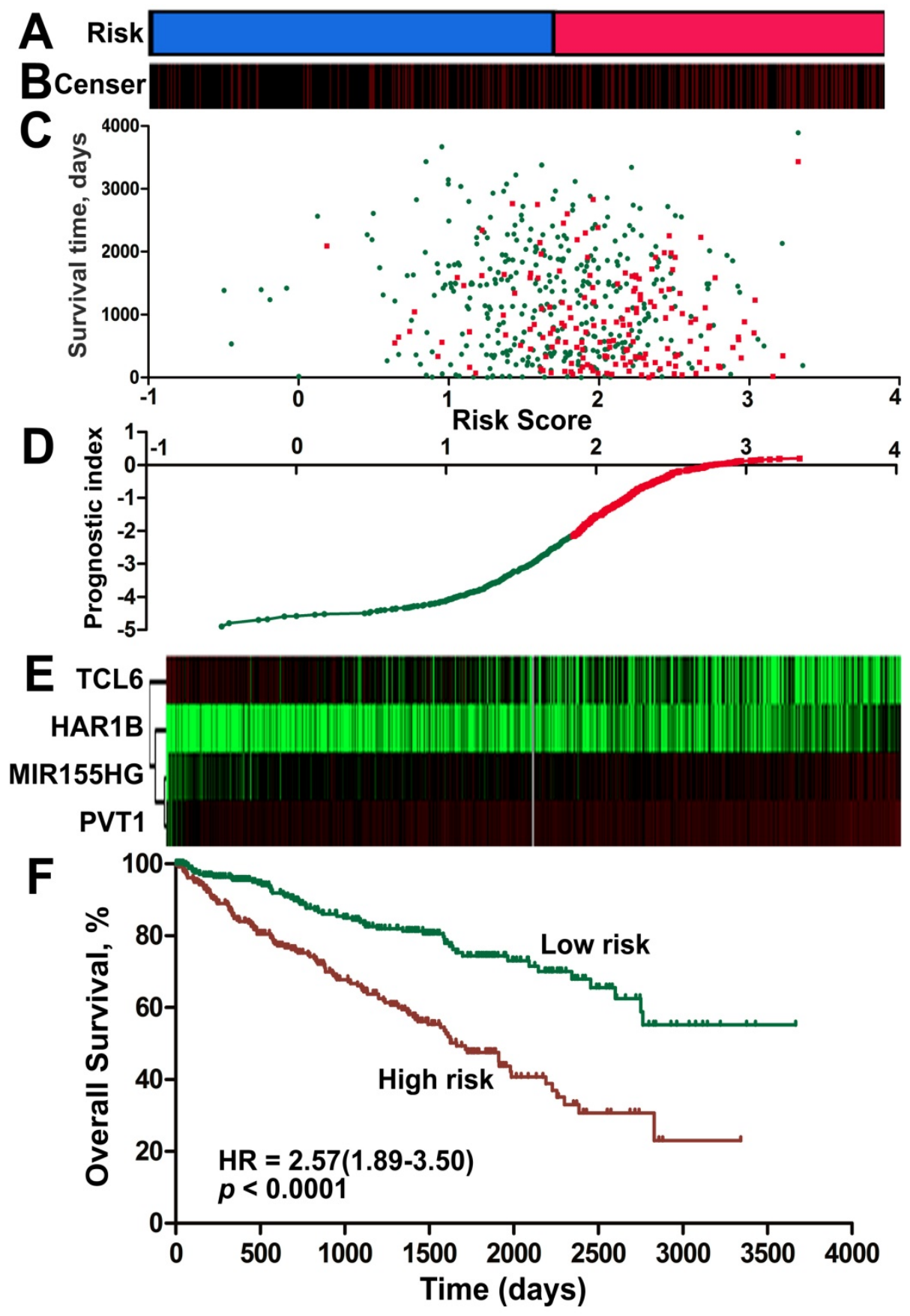

Figure 4. Risk score for the 4-IncRNA signature and outcome in ccRCC patients. Risk score of the IncRNA signature divided into low- and high-score groups. (B) Information related to the censoring event was analyzed. Columns represent ccRCC patients. The black dotted line represents the censored status, and the red line represents patient survival status. (C) Survival status and duration of cases. (D) The IncRNA signature risk score distribution. (E) Heat map of the IncRNA expression profiles. Each row represents a single IncRNA, and each column represents the corresponding patients. The black dotted line represents the median IncRNA risk score cut-off dividing patients into low-risk and high-risk groups. (F) Kaplan-Meier curve for the low-score and high-score group.

Compared with previous studies, our study utilized data from TCGA with high throughput analysis of lncRNAs. A total of 1056 lncRNAs were initially included in the present study, offering a more comprehensive analysis. Furthermore, the significance level was set as 0.001 and the fold change as 1.5 to control the false discovery rate. By combining the 4 identified lncRNAs, the lncRNA signature risk score may act as an independent predictor in ccRCC.
During the training phase, we probed the expression profiles of 60 candidate lncRNAs in ccRCC tissues and adjacent normal kidney tissues; among these, four (TCL6, PVT1, MIR155HG and HAR1B) showed evidently disparate expression between the tissues. TCL6 expression was notably reduced in ccRCC tissues, whereas expression of PVT1, MIR155HG and HAR1B was increased, and this trend was in accordance with previous studies $[18,19]$. 

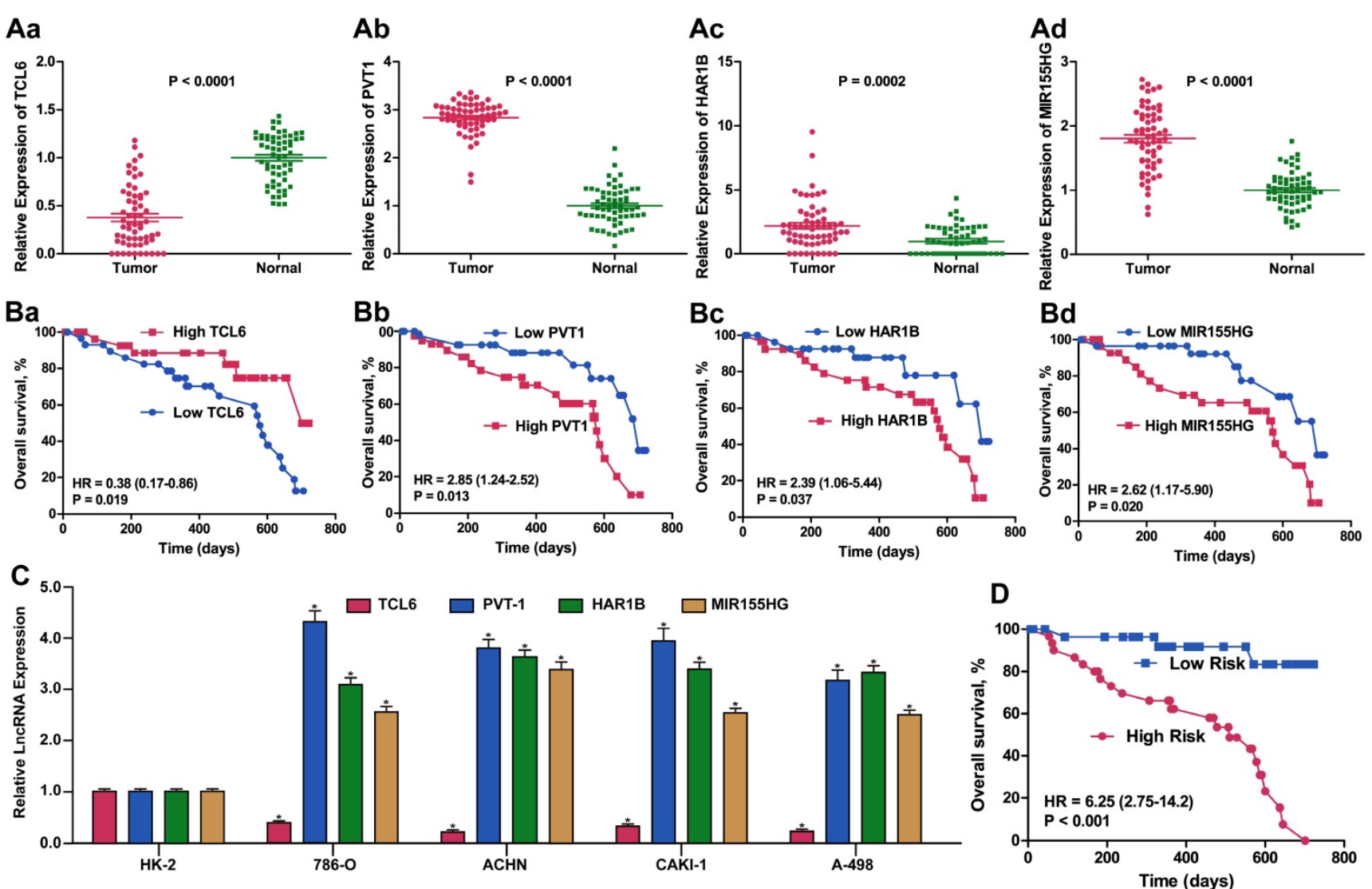

Figure 5. Expression and Kaplan-Meier OS analysis of the 4-IncRNA signature in our validation cohort of ccRCC patients. Relative fold change of validated expression of the four IncRNAs in the Tongji ccRCC cohort compared with normal adjacent tissue determined by qPCR. (B). Kaplan-Meier survival analysis of the four IncRNAs in the Tongji ccRCC validation cohort. (C) Relative fold change of expression of the four IncRNAs in 786-O, ACHN, CAKI-1 and A498 ccRCC cell lines compared with the immortalized proximal tubule epithelial cell line HK2, as determined by qPCR. (D). Kaplan-Meier survival analysis of the 4-IncRNA signature in the Tongji ccRCC validation cohort.

Table 1. Univariate and multivariate Cox proportional hazards analyses of overall survival for patients with ccRCC in TCGA.

\begin{tabular}{|c|c|c|c|c|c|c|c|}
\hline \multirow[t]{2}{*}{ Variables } & \multirow[t]{2}{*}{ Categories } & \multicolumn{3}{|c|}{ Univariate Analysis } & \multicolumn{3}{|c|}{ Multivariate Analysis } \\
\hline & & HR & $95 \% \mathrm{CI}$ & P Value & HR & $95 \% \mathrm{CI}$ & P Value \\
\hline Age & $<65$ vs. $\geq 65$ ys & 1.014 & $1.024-1.058$ & $<0.001$ & 1.031 & $1.015-1.047$ & $<0.001$ \\
\hline Sex & Male vs. female & 0.863 & $0.589-1.266$ & 0.452 & 0.976 & $0.691-1.381$ & 0.892 \\
\hline Laterality & Left vs. Right & 1.618 & $1.117-2.343$ & 0.011 & 1.185 & $0.851-1.649$ & 0.315 \\
\hline Dimension & $<1.5$ vs. $\geq 1.5 \mathrm{~cm}$ & 1.730 & $1.296-2.310$ & $<0.001$ & 0.842 & $0.659-1.076$ & 0.17 \\
\hline tumor stage & T4vs.T3vs.T2vs.T1 & 2.377 & $1.986-2.846$ & $<0.001$ & 1.564 & $1.238-1.977$ & $<0.001$ \\
\hline tumor grade & G4vs.G3vs.G2vs.G1 & 1.322 & $0.897-1.365$ & 0.236 & 1.127 & $0.907-1.4$ & 0.279 \\
\hline Lymph node & Negative vs. positive & 0.805 & $0.667-0.971$ & 0.024 & 0.841 & $0.707-1$ & 0.05 \\
\hline Metastasis & Yes vs. No & 2.231 & $1.579-3.152$ & $<0.001$ & 1.636 & $0.957-2.797$ & 0.072 \\
\hline TCL6 & High vs. Low & 0.484 & $0.357-0.658$ & $<0.001$ & 0.947 & $0.878-1.023$ & 0.166 \\
\hline MIR155HG & High vs. Low & 1.763 & $1.298-2.394$ & 0.0003 & 1.065 & 0.949-1.195 & 0.282 \\
\hline HAR1B & High vs. Low & 1.564 & $1.143-2.129$ & 0.0051 & 1.211 & $1.016-1.443$ & 0.032 \\
\hline PVT1 & High vs. Low & 1.791 & $1.318-2.434$ & 0.0002 & 1.155 & $0.938-1.422$ & 0.175 \\
\hline Risk Score & High vs. Low & 2.57 & $1.89-3.5$ & $<0.001$ & 1.932 & $1.392-2.682$ & $<0.001$ \\
\hline
\end{tabular}

The T-cell leukemia/lymphoma 6 (TCL6) locus maps $7 \mathrm{~kb}$ centromeric to the TML1 locus and is composed of at least 12 exons with small alternative exons [20]. Similar to our results, Su et al. reported that TCL6 was overexpressed in RCC; decreased TCL6 expression suggested an inferior prognosis for patients with ccRCC. Furthermore, overexpression of TCL6 in 786-O and Caki-1 RCC cells decreased proliferation and increased apoptosis compared to controls [19].
PVT1 is a widely reported oncogene that may be involved in renal cancer, lung cancer, colorectal cancer, gastric cancer, hepatocellular carcinoma, ovarian cancer, and leukaemia [21-24]. Among all cancer types, renal clear cell carcinoma displays the strongest upregulation of PVT1, and its misregulation in cCRCC is largely associated with promoter hypomethylation [18]. Indeed, Wang et al. reported that PVT1 overexpression in hepatocellular carcinoma cells enhanced cell proliferation, cell cycling, and the 
acquisition of stem cell-like properties by stabilizing NOP2, suggesting that it may act as an oncogene in ccRCC progression [25]. Xu et al. also reported that high expression of PVT1 predicted an inferior prognosis in patients with gastric cancer. By interacting with FOXM, prominent expression of PVT1 enhanced cancer proliferation and invasion [24]. A serum 5-lncRNA signature that includes PVT1 was recently discovered as a biomarker facilitating the detection of ccRCC [26]. Consistent with our results, Posa et al. found PVT1 to serve as a prognostic factor for novel therapeutic interventions in RCC [18].

The miR-155 host gene (MIR155HG), which is located on chromosome 21, encodes miR-155 [27]; miR-155 expression has been confirmed to be up-regulated in ccRCC tissue and cell lines, and it may function as an oncogene by targeting $\mathrm{BACH} 1$ [28, 29]. Recent studies have provided evidence that MIR155HG mainly influences B-cell receptor signaling and is associated with aggressive disease in leukaemia [30]. Furthermore, Wang et al. reported that MIR155HG, as one of a 4-lncRNA signature, had prognostic value for anaplastic glioma and was increased with tumor grade [31]. We found both HAR1A and HAR1B to be up-regulated in ccRCC and associated with poor survival. Similar to our results, a 9-lncRNA signature consisting of HAR1A and its upregulation predicted breast cancer recurrence and served as a prognostic marker for breast cancer [32]. Using TCGA, Ma et al. investigated lncRNAs as prognostic biomarkers for papillary thyroid cancer, revealing that low HAR1A expression was associated with cancer recurrence and poor prognosis [33]. Regardless, there have been no reports on MIR155HG and HAR1B in ccRCC to date. Therefore, exploring their roles in tumorigenesis may contribute to demonstrating their oncogenic or suppressor function in ccRCC patients.

No comprehensive analysis to investigate lncRNA profiling in RCC has been performed thus far. However, the results of our study are helpful for exploration of potential lncRNA biomarkers in human ccRCC. We suggest four promising lncRNAs that have been abundantly reported as having altered expression and significant dysregulation. Nonetheless, this panel is only specific for ccRCC. Furthermore, some limitations existed in our study that should be considered. First, it should be noted that a large portion of unknown lncRNAs were missing due to the intrinsic limitation of the microarray technique and probe repurposing method. Second, only the dataset (KIRC) from TCGA and our ccRCC cohort were evaluated in this research, resulting in inadequate samples for the 4-lncRNA signature model of prognosis. Accordingly, further studies with larger cohorts are warranted to validate our prognostic model. Finally, ccRCC covers most of RCC cases. When validating our prognostic model in kidney renal papillary cell carcinoma (KIRP) and kidney chromophobe carcinoma (KIRC), the results showed no significant difference. Thus, we suggest that the significant prognostic value of the lncRNA signature be further assessed in other subtypes of RCC.

\section{Conclusions}

In summary, by analyzing the genome-wide lncRNA expression profiles from TCGA, a panel of four IncRNAs was identified that may serve as an independent predictor of prognosis in ccRCC. With further confirmation of the mechanisms by which these lncRNAs impact ccRCC progression, the 4-lncRNA signature might not only have prognostic value for low-risk patients who will benefit from nephroectomy but may also provide a deeper understanding of the molecular heterogeneity of ccRCC. Therefore, more research is needed to uncover novel diagnostic or prognostic lncRNA candidates and elucidate their function in ccRCC.

\section{Supplementary Material}

Supplementary figures and tables. http://www.jcancer.org/v11p4274s1.pdf

\section{Acknowledgements}

This project was supported by the National Natural Science Foundation of China (81270788, 81470935, 81370805, 81402098, 81402087, 31372562, $81670645,81602236)$, the Chenguang Program of Wuhan Science and Technology Bereau (201507040 4010199, 2015071704021644), the National Major Scientific and Technological Special Project for Significant New Drugs Development (2012ZX09 303018), and the National High Technology Research and Development Program 863 (2014AA020607). Kun Tang wants to thank the invaluable support received from his wife Mint over the years.

We would also like to thank all participants enrolled in the present study.

\section{Competing Interests}

The authors have declared that no competing interest exists.

\section{References}

1. Siegel RL, Miller KD, Jemal A. Cancer statistics, 2018. CA Cancer J Clin. 2018; 68: 7-30.

2. Perazella MA, Dreicer R, Rosner MH. Renal cell carcinoma for the nephrologist. Kidney Int. 2018; 94: 471-483.

3. Martínez-Salamanca JI, Huang WC, Millán I, et al. Prognostic impact of the 2009 UICC/AJCC TNM staging system for renal cell carcinoma with venous extension. Eur Urol. 2011; 59: 120-127. 
4. Posadas EM, Limvorasak S, Figlin RA. Targeted therapies for renal cell carcinoma. Nat Rev Nephrol. 2017; 13: 496-511.

5. Jiang S, Cheng SJ, Ren LC, et al. An expanded landscape of human long noncoding RNA. Nucleic Acids Res. 2019.

6. Yu G, Yao W, Wang J, et al. LncRNAs expression signatures of renal clear cell carcinoma revealed by microarray. PLoS One. 2012; 7: e42377.

7. Ulitsky I, Bartel DP. lincRNAs: genomics, evolution, and mechanisms. Cell. 2013; 154: 26-46.

8. Evans JR, Feng FY, Chinnaiyan AM. The bright side of dark matter: IncRNAs in cancer. J Clin Invest. 2016; 126: 2775-2782.

9. Shi $\mathrm{D}, \mathrm{Qu} \mathrm{Q}, \mathrm{Chang} \mathrm{Q}$, et al. A five-long non-coding RNA signature to improve prognosis prediction of clear cell renal cell carcinoma. Oncotarget. 2017; 8: 58699-58708

10. Zhu H, Lu J, Zhao H, et al. Functional Long Noncoding RNAs (lncRNAs) in Clear Cell Kidney Carcinoma Revealed by Reconstruction and Comprehensive Analysis of the lncRNA-miRNA-mRNA Regulatory Network. Med Sci Monit. 2018; 24: 8250-8263.

11. Schmitt AM, Chang HY. Long Noncoding RNAs in Cancer Pathways. Cancer Cell. 2016; 29: 452-463

12. Vallot C, Rougeulle C. Long non-coding RNAs and human X-chromosome regulation: a coat for the active $X$ chromosome. RNA Biol. 2013; 10: 1262-1265.

13. Sun $\mathrm{W}$, Yang $\mathrm{Y}, \mathrm{Xu} \mathrm{C}$, et al. Regulatory mechanisms of long noncoding RNAs on gene expression in cancers. Cancer Genet. 2017; 216-217: 105-110.

14. Brooks SA, Brannon AR, Parker JS, et al. ClearCode34: A prognostic risk predictor for localized clear cell renal cell carcinoma. Eur Urol. 2014; 66: 77-84.

15. Rini B, Goddard A, Knezevic D, et al. A 16-gene assay to predict recurrence after surgery in localised renal cell carcinoma: development and validation studies. Lancet Oncol. 2015; 16: 676-685.

16. Liu T, Sui J, Zhang Y, et al. Comprehensive analysis of a novel lncRNA profile reveals potential prognostic biomarkers in clear cell renal cell carcinoma. Oncol Rep. 2018; 40: 1503-1514.

17. Blondeau JJ, Deng $\mathrm{M}$, Syring $\mathrm{I}$, et al. Identification of novel long non-coding RNAs in clear cell renal cell carcinoma. Clin Epigenetics. 2015; 7: 10.

18. Posa I, Carvalho S, Tavares J, et al. A pan-cancer analysis of MYC-PVT1 reveals CNV-unmediated deregulation and poor prognosis in renal carcinoma. Oncotarget. 2016; 7: 47033-47041.

19. Su H, Sun T, Wang H, et al. Decreased TCL6 expression is associated with poor prognosis in patients with clear cell renal cell carcinoma. Oncotarget. 2017; 8: 5789-5799.

20. Saitou M, Sugimoto J, Hatakeyama T, et al. Identification of the TCL6 genes within the breakpoint cluster region on chromosome 14q32 in T-cell leukemia. Oncogene. 2000; 19: 2796-2802.

21. Takahashi Y, Sawada G, Kurashige J, et al. Amplification of PVT-1 is involved in poor prognosis via apoptosis inhibition in colorectal cancers. Br J Cancer. 2014; 110: 164-171.

22. Grampp S, Platt JL, Lauer V, et al. Genetic variation at the $8 \mathrm{q} 24.21$ renal cancer susceptibility locus affects HIF binding to a MYC enhancer. Nat Commun. 2016; 7: 13183

23. Martini P, Paracchini L, Caratti G, et al. IncRNAs as Novel Indicators of Patients' Prognosis in Stage I Epithelial Ovarian Cancer: A Retrospective and Multicentric Study. Clin Cancer Res. 2017; 23: 2356-2366.

24. Xu MD, Wang $Y$, Weng $W$, et al. A Positive Feedback Loop of IncRNA-PVT1 and FOXM1 Facilitates Gastric Cancer Growth and Invasion. Clin Cancer Res. 2017; 23: 2071-2080.

25. Wang F, Yuan JH, Wang SB, et al. Oncofetal long noncoding RNA PVT1 promotes proliferation and stem cell-like property of hepatocellular carcinoma cells by stabilizing NOP2. Hepatology. 2014; 60: 1278-1290.

26. Wu Y, Wang YQ, Weng WW, et al. A serum-circulating long noncoding RNA signature can discriminate between patients with clear cell renal cell carcinoma and healthy controls. Oncogenesis. 2016; 5: e192.

27. Vargova K, Curik N, Burda $\mathrm{P}$, et al. MYB transcriptionally regulates the miR-155 host gene in chronic lymphocytic leukemia. Blood. 2011; 117: 3816-3825.

28. Li S, Chen T, Zhong Z, et al. microRNA- 155 silencing inhibits proliferation and migration and induces apoptosis by upregulating BACH1 in renal cancer cells. Mol Med Rep. 2012; 5: 949-954.

29. Tang $\mathrm{K}, \mathrm{Xu} \mathrm{H}$. Prognostic value of meta-signature miRNAs in renal cell carcinoma: an integrated miRNA expression profiling analysis. Sci Rep. 2015; 5: 10272 .

30. Cui B, Chen L, Zhang S, et al. MicroRNA-155 influences B-cell receptor signaling and associates with aggressive disease in chronic lymphocytic leukemia. Blood. 2014; 124: 546-554.

31. Wang W, Yang F, Zhang L, et al. LncRNA profile study reveals four-lncRNA signature associated with the prognosis of patients with anaplastic gliomas. Oncotarget. 2016; 7: 77225-77236.

32. Liu H, Li J, Koirala P, et al. Long non-coding RNAs as prognostic markers in human breast cancer. Oncotarget. 2016; 7: 20584-20596.

33. Ma B, Liao T, Wen D, et al. Long intergenic non-coding RNA 271 is predictive of a poorer prognosis of papillary thyroid cancer. Sci Rep. 2016; 6: 36973 